\title{
Value of Ultrasound for Stability Assessment of Isolated Lateral Malleolar Fractures Compared to Stress Radiography and Arthroscopy
}

\author{
Hyong Nyun Kim, MD, Seungmin Jeong, MD*, Young Uk Park, MD* \\ Department of Orthopedic Surgery, Kangnam Sacred Heart Hospital, Hallym University College of Medicine, Seoul, \\ ${ }^{*}$ Department of Orthopedic Surgery, Ajou University Hospital, Ajou University School of Medicine, Suwon, Korea
}

Background: This study was performed to evaluate the value of using ultrasound for stability assessment of isolated lateral malleolar fractures compared to simple X-ray, stress radiography, and arthroscopy.

Methods: This is a prospective cohort study with 25 consecutive patients who underwent an arthroscopic examination and subsequent surgery for isolated lateral malleolar ankle fractures. Before operation, simple and external rotation stress radiographs were obtained. Ultrasound was performed to assess the medial deltoid ligament prior to operation. Arthroscopic findings were used as reference standards. Sensitivity, specificity, and positive and negative predictability were calculated and compared using receiver operating characteristic (ROC) curve analysis for simple radiography, stress radiography, and ultrasound examination.

Results: The sensitivity and specificity of ultrasound for tears of the deep deltoid ligament were $94.74 \%$ and $66.67 \%$, respectively. But, they were both $100 \%$ for complete tears of the ligament. ROC curve analysis showed that the ultrasound examination was significantly more accurate than simple and stress radiography.

Conclusions: Ultrasound could be used to assess the instability of isolated lateral malleolar fractures. Commonly used stress radiography appears to overrate the need for operative treatment.

Keywords: Ankle fracture, Isolated lateral malleolar fracture, Stability, Ultrasound

Ankle fractures are commonly treated orthopedic trauma injuries. There is no doubt about surgical treatment of unstable bimalleolar or trimalleolar ankle fractures. However, treatment of isolated lateral malleolar fractures remains controversial. ${ }^{1-6)}$ The treatment strategy depends on the stability of the ankle joint. Conceptualizing the ankle joint as a ring, like the pelvis, is helpful in deciding which fractures are stable or unstable. One fracture in the otherwise intact ring is stable. ${ }^{7)}$ Therefore, pure isolated lateral malleolar fractures are stable. However, combined medial deltoid ligament injury will make the ankle unstable. The

Received January 31, 2020; Accepted April 27, 2020

Correspondence to: Young Uk Park, MD

Department of Orthopedic Surgery, Ajou University Hospital, 164 World

cup-ro, Yeongtong-gu, Suwon 16499, Korea

Tel: +82-31-219-5220, Fax: +82-31-219-5229

E-mail: parkyounguk@gmail.com deep deltoid ligament is recognized as a primary stabilizer of the ankle. It prevents lateral talar shift and external rotation (ER) of the talus. ${ }^{6}$ Given that the inability to maintain anatomic alignment of the mortise has been correlated with poor outcomes, the assessment of stability is critical.

Ankle stability has been traditionally assessed through clinical findings and simple radiography. If medial tenderness, swelling, and ecchymosis are present on clinical examination in combination with lateral talar shift on simple radiography, a medial deltoid ligament injury is suspected. However, clinical findings alone are insufficient to determine the status of the medial deltoid ligament; other objective assessment methods are therefore needed.

In clinical evaluation of lateral malleolar fractures with medial tenderness, which appear to be stable on plain radiography, stress radiography is used to determine medial deltoid injury and stability of the ankle. The me- 
Kim et al. Value of Ultrasound for Stability Assessment of Isolated Lateral Malleolar Fractures

Clinics in Orthopedic Surgery • Vol. 12, No. 4, $2020 \bullet$ www.ecios.org

dial clear space (MCS), which is defined as the distance between the lateral border of the medial malleolus and the medial border of the talus at the level of the talar dome, ${ }^{8)}$ is an indicator of the deep deltoid ligament. ER stress test is a popular method for assessing ankle mortise stability. ${ }^{2-5,9)}$ However, it has several problems. It is difficult to make the correct ankle position. Different examiners produce various amounts of force. It can cause pain and further injury. The examiner is at risk of radiation hazards. Gravity stress radiography has also been used to assess ankle stability. It is known to be better than ER stress radiography. However, it might give false-negative results. ${ }^{10)}$

Magnetic resonance imaging (MRI) and arthroscopy can be used for stability assessment. ${ }^{2,11,12)}$ However, the practical use of these modalities is limited because of their high cost and invasiveness. Ultrasound (US) is increasingly used for detection of musculoskeletal soft tissue injuries. For the foot and ankle, US is quite useful because most tendons, ligaments, and nerves are located just beneath the skin. Furthermore, US examination can be performed during the first visit of a patient. It is also likely to be more accurate than the traditional method of palpating ligaments to diagnose a possible injury. ${ }^{13)}$ Chen et al. ${ }^{14)}$ have reported its use for stability assessment of isolated lateral malleolar ankle fractures. However, to the best of our knowledge, a comparison study between US and other diagnostic tools for stability assessment has not been reported yet. Our hypothesis was that US could be used for more accurate assessment of isolated lateral malleolar ankle fractures. Therefore, the objective of this study was to evaluate the value of using US for stability assessment of isolated lateral malleolar fractures compared to simple X-ray, stress radiography, and arthroscopy. We investigated the accuracy of US examination in comparison with simple radiography and ER stress radiography and arthroscopy for diagnosing instability of the ankle in isolated lateral malleolar ankle fractures.

\section{METHODS}

This study was approved by Institutional Review Board of Ajou University Hospital (IRB No. AJIRB-MED-MDB16-323). Informed consent for enrollment to the study was obtained from each patient. Patients aged at least 19 years with isolated lateral malleolar ankle fractures of LaugeHansen Supination-External Rotation (SER) type, who were treated from May 2016 to May 2017 at our institution, were eligible for inclusion in the present prospective study. Patients with bilateral ankle fractures, pathologic fractures, previous ankle injuries, concomitant tibial shaft fractures, peripheral neuropathy, soft-tissue infection in the region of the ankle, generalized inflammatory arthritis, or inability to walk unaided before injury were excluded. Fractures were classified by a senior foot and ankle surgeon (YUP), who were responsible for each patient's care. Of 37 patients who were screened for inclusion, 12 were excluded due to concomitant tibial shaft fractures $(n=5)$, previous ankle injuries $(\mathrm{n}=4)$, peripheral neuropathy $(\mathrm{n}=$ $2)$, soft tissue infection $(n=1)$, or generalized arthritis $(n=$ 1). Finally, 25 patients were enrolled, including 11 women and 14 men, whose mean age was $42.2 \pm 15.9$ years.

Before the radiographic examination, swelling, tenderness, and ecchymosis on the medial deltoid area were assessed for each patient. All patients had tenderness and swelling over the medial deltoid area. Stability was assessed on initial simple radiography. Each result was recorded as positive when the MCS was $\geq 5 \mathrm{~mm}$, tibiofibular overlap was $<5 \mathrm{~mm}$, tibiofibular clear space was $\geq 10 \mathrm{~mm}$, or sideto-side difference was $\geq 1 \mathrm{~mm}$. These measurements were adjusted for magnification using a constant source-todetector distance of $115 \mathrm{~cm}$ and a standard $30 \mathrm{~mm}$ radiographic marker. ${ }^{10)}$ Two authors (SYL, YUP) measured each distance and calculated interobserver reliability. Disagreements were resolved through consensus discussion.

For cases with negative simple radiography, manual ER stress radiography ${ }^{15,16)}$ was performed by one senior foot and ankle surgeon (YUP) or a senior orthopedic foot and ankle resident (Orthopedic residents of Ajou University Hospital), who completed foot and ankle training. A total of 4 surgeons (YUP, 3 residents) were involved in the ER stress test. Each result was recorded as positive when the MCS was $\geq 5 \mathrm{~mm}$ or side-to-side difference was $\geq 1$ $\mathrm{mm}$. Positive cases on either simple or stress radiography were believed to be unstable ankle fractures. Operative treatment was then decided for those patients.

Before surgery, all patients underwent an US examination. They were evaluated by a musculoskeletal radiologist (SHP), who had more than 10 years of experience as a diagnostician in musculoskeletal imaging. It was performed with a Logiq 7 unit (LOGIQ P6; GE Healthcare, Chicago, IL, USA) and a linear probe. Each patient was in a sitting position with one leg placed on the examination table. The transducer was placed over the medial deltoid ligament. The transducer scanned the deltoid ligament in a fan-shaped fashion in the oblique coronal plane. A normal deltoid ligament appeared as a hyperechoic band with 4 to $5 \mathrm{~mm}$ in thickness, connecting the medial malleolus to the posterior talus, sustentaculum tali, navicular, and anterior talus (Fig. 1). Criteria for a complete rupture of the deltoid ligament were discontinuity and direct contact of the pos- 
Kim et al. Value of Ultrasound for Stability Assessment of Isolated Lateral Malleolar Fractures

Clinics in Orthopedic Surgery • Vol. 12, No. 4, $2020 \bullet$ www.ecios.org

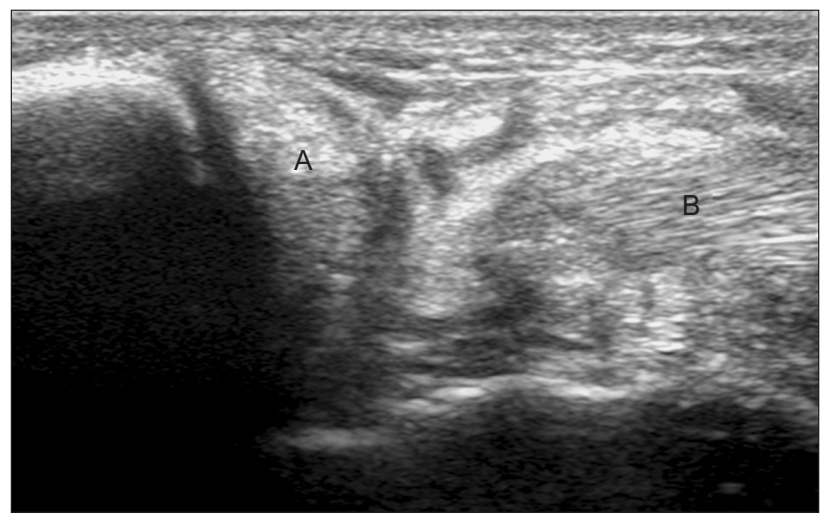

Fig. 1. Complete rupture of the deltoid ligament was diagnosed if discontinuity $(A)$ and direct contact of the posterior tibial tendon to the expanded articular pouch (B) were observed.

terior tibial tendon to the expanded articular pouch (Fig. 2). Cases with vague findings (echo failing to match complete tear or intact ligament) were regarded as partial tears.

After surgical fixation of the lateral malleolar fracture (interfragmentary screw fixation and lateral plating), the arthroscopic examination was performed by a single senior surgeon (YUP). The ankle joint was visualized through standard anterolateral and anteromedial portals. A systematic arthroscopic examination was performed to visualize internal structures (deltoid ligament, medial gutter, talus, talofibular articulation, lateral gutter, anterior inferior tibiofibular ligament, anterior talofibular ligament, and calcaneofibular ligament) in order to clarify injury of the medial deltoid ligament. Ligaments were inspected visually, and photographs were taken with the foot internally and externally rotated while the mid tibial shaft was stabilized. The deltoid ligament complex was manually stressed intraoperatively by applying ER forces to the foot. The integrity of the deltoid ligament was classified as intact, partially ruptured, or completely ruptured. ${ }^{11)}$

The sample size was determined using an area under the receiver operating characteristic (ROC) curve based on a pilot study. Our hypothesis was that US was an accurate method for assessing tears of the deep deltoid ligament. The sample size was determined by assuming an area under curve of 1.00 for complete tear and 0.807 for total injury of the deltoid ligament. We calculated a sample size of 8 patients for complete tear and 24 patients for deltoid injury in total (partial or complete tear). This would permit a type- 1 error rate of 0.05 , with a type- 2 error rate of 0.20 .

Sensitivity, specificity, and positive and negative predictability were calculated for simple radiography, stress radiography, and US examination, using the arthroscopic

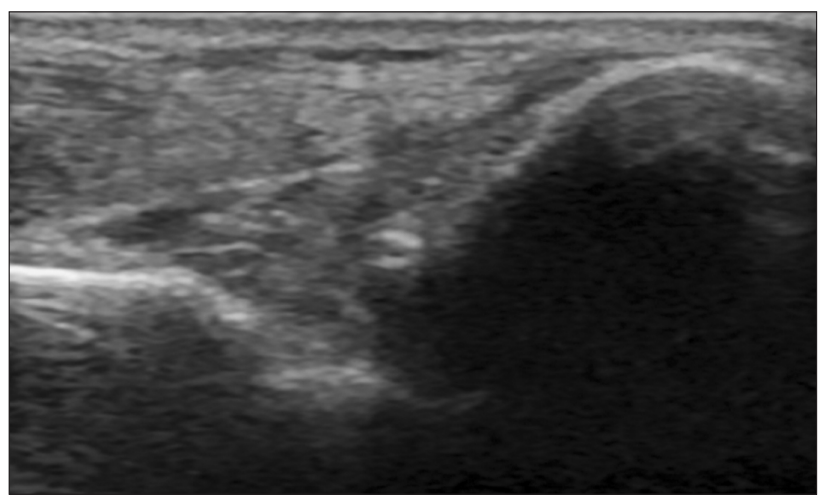

Fig. 2. The normal deltoid ligament appeared as a 4 to 5-mm-thick hyperechoic band, connecting the medial malleolus to the posterior talus, sustentaculum tali, navicular, and anterior talus.

findings as a reference. ROC curve analysis was used to calculate associations between simple and stress radiography and US examination as a reference to arthroscopic findings. A $p$-value $<0.05$ was considered statistically significant.

\section{RESULTS}

A total of 13 patients had unstable ankle fractures on simple radiography and 12 patients on stress radiography (Table 1). On US, 7 patients had complete ruptures of the deltoid ligament (Fig. 1). Thirteen patients showed partial tears while 5 patients had normal deltoid ligaments on US (Fig. 2). On arthroscopy, 7 patients had complete ruptures of the deltoid ligament. Twelve patients showed partial tears while 6 patients had normal deltoid ligaments on arthroscopy (Fig. 3).

The sensitivity and specificity of US for tears of the deep deltoid ligament were $94.7 \%$ and $66.7 \%$, respectively. But, they were both $100 \%$ for complete tears of it (Table 2). The ROC curve analysis showed that US examination was significantly more accurate than simple and stress radiography (Table 3).

\section{DISCUSSION}

The most important finding of this study was that most patients did not have complete deltoid ligament injury. All patients were diagnosed as having unstable ankle fractures by simple or stress radiography. However, many of them did not have severely injured deltoid ligaments based on arthroscopic findings.

The MCS, which was defined as the distance between the lateral border of the medial malleolus and the 
Kim et al. Value of Ultrasound for Stability Assessment of Isolated Lateral Malleolar Fractures

Clinics in Orthopedic Surgery • Vol. 12, No. 4, $2020 \bullet$ www.ecios.org

\section{Table 1. Details of the Total Cases}

\begin{tabular}{|c|c|c|c|c|c|c|}
\hline No. & $\begin{array}{l}\text { Age } \\
(\mathrm{yr})\end{array}$ & Sex & $\begin{array}{l}\text { Simple } \\
\text { X-ray }\end{array}$ & $\begin{array}{l}\text { Stress } \\
\text { X-ray }\end{array}$ & $\begin{array}{c}\text { US } \\
\text { Deltoid }\end{array}$ & $\begin{array}{c}\text { AS } \\
\text { Deltoid }\end{array}$ \\
\hline 1 & 57 & $\mathrm{~F}$ & + & & 0 & 0 \\
\hline 2 & 56 & $\mathrm{~F}$ & + & & 1 & 0 \\
\hline 3 & 19 & $\mathrm{~F}$ & + & & 1 & 1 \\
\hline 4 & 44 & $\mathrm{~F}$ & + & & 1 & 1 \\
\hline 5 & 49 & $\mathrm{~F}$ & + & & 0 & 1 \\
\hline 6 & 28 & $M$ & + & & 1 & 1 \\
\hline 7 & 45 & $M$ & - & + & 2 & 2 \\
\hline 8 & 22 & $M$ & + & & 1 & 0 \\
\hline 9 & 24 & $\mathrm{M}$ & + & & 2 & 2 \\
\hline 10 & 59 & $\mathrm{~F}$ & - & + & 0 & 0 \\
\hline 11 & 70 & $\mathrm{M}$ & - & + & 2 & 2 \\
\hline 12 & 45 & $M$ & - & + & 1 & 1 \\
\hline 13 & 23 & $M$ & + & & 2 & 2 \\
\hline 14 & 40 & $M$ & + & & 1 & 1 \\
\hline 15 & 56 & $\mathrm{~F}$ & - & + & 1 & 1 \\
\hline 16 & 50 & $\mathrm{~F}$ & - & + & 0 & 0 \\
\hline 17 & 55 & $\mathrm{~F}$ & + & & 0 & 0 \\
\hline 18 & 21 & $\mathrm{M}$ & - & + & 1 & 1 \\
\hline 19 & 49 & $\mathrm{~F}$ & - & + & 1 & 1 \\
\hline 20 & 27 & $M$ & + & & 1 & 1 \\
\hline 21 & 67 & M & + & & 1 & 1 \\
\hline 22 & 49 & $M$ & - & + & 2 & 2 \\
\hline 23 & 40 & $\mathrm{M}$ & - & + & 1 & 1 \\
\hline 24 & 19 & $M$ & - & + & 2 & 2 \\
\hline 25 & 55 & $\mathrm{~F}$ & - & + & 2 & 2 \\
\hline
\end{tabular}

US: ultrasound, AS: arthroscopy, 0: intact, 1: partial tear, 2: complete tear.

medial border of the talus at the level of the talar dome, has been considered as an indicator of the status of the deep deltoid ligament. ${ }^{9)}$ An MCS of more than $5 \mathrm{~mm}$ on simple or stress radiography suggests a total tear of the deep deltoid ligament. It is believed to be an indication for operative treatment. ${ }^{1,2,9,10,17,18)}$ Recently, several studies have raised doubts about the accuracy of the MCS for predicting injury of deep deltoid ligaments. ${ }^{6,911,17,19-21)}$ Koval et al. ${ }^{9)}$ have reported that MCS measurements on stress radiographic testing do not correlate with MRI findings of deep

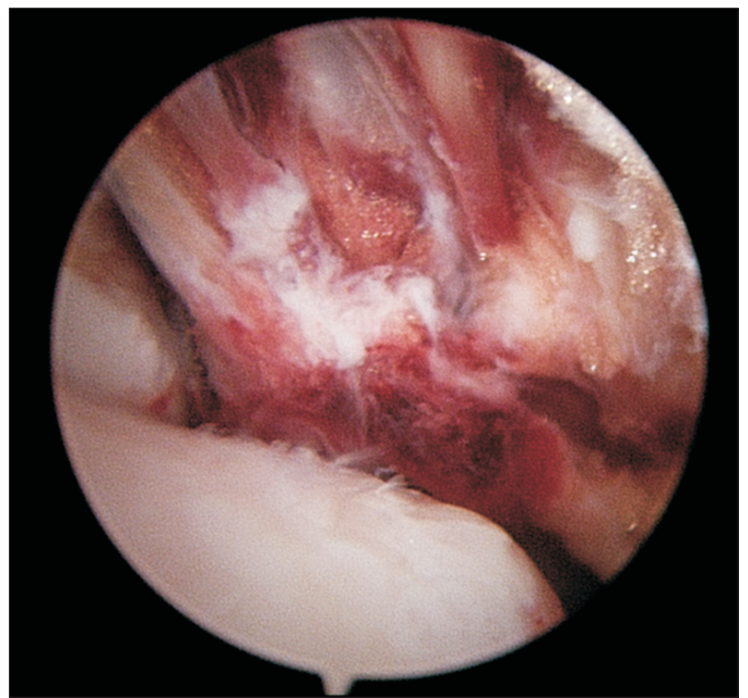

Fig. 3. Complete rupture of a deltoid ligament on arthroscopy. Loss of continuity and the hemorrhagic torn end of the ligament were seen. The arthroscope inserted through the anteromedial portal to the posterior joint space could go into the posteromedial joint space without difficulty.

deltoid rupture. Schuberth et al. ${ }^{11)}$ have also stated that a widened MCS is overemphasized as an indicator of deltoid integrity in their arthroscopic study. They have pointed out that an accurate diagnosis of deltoid rupture by noninvasive methods remains a clinical challenge.

MRI has been used to diagnose soft tissue injury of ankle fractures. ${ }^{1,12,22-24)} \operatorname{Vogl}$ et al. ${ }^{24)}$ have reported that MRI is a highly sensitive and specific tool for the evaluation of syndesmotic injury. Nielson et al. ${ }^{23)}$ have reported that the level of fibula fractures does not correlate with the integrity of interosseous membrane injury. However, there are limited studies about its use for stability assessment of isolated lateral malleolar fractures. Koval et al. ${ }^{9)}$ found that $19(90 \%)$ of 21 patients had evidence of partially torn deep deltoid ligaments on MRI. These patients were treated nonoperatively. However, 2 patients with complete deep deltoid injury on MRI underwent surgery. They stated that all fractures united without evidence of residual MCS widening or posttraumatic joint space narrowing. ${ }^{9)}$ However, Nortunen et al. ${ }^{1)}$ found that all patients had an injury involving the deep deltoid ligament on MRI (an edematous ligament, a partial tear, and a total tear) regardless of the degree of MCS, even though the MCS was increased $(p<$ 0.001 ) according to the severity of deep deltoid ligament injury based on MRI. They reported that interobserver agreement of the ER stress test was excellent, whereas the interobserver reliability of the MRI assessment was fair to moderate. Therefore, they did not recommend the use of MRI when choosing between the operative and nonopera- 
Kim et al. Value of Ultrasound for Stability Assessment of Isolated Lateral Malleolar Fractures

Clinics in Orthopedic Surgery • Vol. 12, No. 4, $2020 \bullet$ www.ecios.org

Table 2. Sensitivity and Specificity of Simple/Stress Radiography and Ultrasound for Deltoid Ligament Injury

\begin{tabular}{|c|c|c|c|c|}
\hline Variable & Sensitivity (\%) & Specificity (\%) & +Predictability (\%) & -Predictability (\%) \\
\hline \multicolumn{5}{|l|}{ Complete tear } \\
\hline Simple X-ray & 28.57 & 38.89 & 15.38 & 58.33 \\
\hline Stress X-ray & 50.00 & 50.00 & 50.00 & 50.00 \\
\hline Ultrasound & 100.00 & 100.00 & 100.00 & 100.00 \\
\hline \multicolumn{5}{|c|}{ Injury in total (partial or complete) } \\
\hline Simple X-ray & 47.37 & 33.33 & 69.23 & 16.67 \\
\hline Stress X-ray & 100.00 & 50.00 & 83.33 & 100.00 \\
\hline Ultrasound & 94.74 & 66.67 & 90.00 & 80.00 \\
\hline
\end{tabular}

Table 3. Comparison of ROC Curves of Simple/Stress Radiography and Ultrasound for Tears of the Deep Deltoid Ligament

\begin{tabular}{llllc}
\multicolumn{1}{c}{ Variable } & AUC & SE & $95 \% \mathrm{Cl}$ & $p$-value \\
\hline Complete tear & & & & \\
\hline Simple X-ray & 0.663 & 0.110 & $0.448-0.838$ & 0.138 \\
\hline Stress X-ray & 0.500 & 0.000 & $0.295-0.705$ & 1.00 \\
\hline Ultrasound & 1.000 & 0.000 & $0.863-1.000$ & $<0.001$ \\
\hline Injury in total (partial or complete) & & & & 0.424 \\
\hline Simple X-ray & 0.576 & 0.121 & $0.383-0.786$ & 1.000 \\
\hline Stress X-ray & 0.500 & 0.000 & $0.295-0.705$ & 0.005 \\
\hline Ultrasound & 0.807 & 0.109 & $0.601-0.936$ & \\
\hline
\end{tabular}

ROC: receiver operating characteristic, AUC: area under the curve, SE: standard error, Cl: confidence interval.

tive treatment of SER-type ankle fractures. Regular use of MRI for assessment of ankle fractures also can become a burden for the patient because of its high cost and inconvenience of examination.

Arthroscopy has been used to assess cartilage lesions and ligament injury in acute ankle fractures. ${ }^{25)}$ Schuberth et al. ${ }^{11)}$ used arthroscopy as a standard to compare with the MCS on stress radiography, but the value of arthroscopy in assessing the stability of ankle fractures could not be determined. Arthroscopic evaluation of the deep deltoid ligament in patients with suspected unstable ankle fractures is not practical because of the invasiveness of the diagnostic modality even if it might be an accurate assessment method.

Henari et al. ${ }^{26)}$ performed a similar comparison study between US and arthrography and found that US was a highly accurate diagnostic modality $(100 \%$ sensitivity and specificity) in the assessment of the deltoid liga- ment. Chen et al. ${ }^{14)}$ have also stated that US allows for direct and noninvasive evaluation of the deltoid ligament in patients with isolated lateral malleolar fractures. They confirmed surgical findings for complete rupture cases of the deltoid ligaments. However, they could not prove correlation between sonographic findings and real pathology in nonsurgical patients. ${ }^{14)}$

Recently, Sanders et al. ${ }^{3)}$ published similar functional results between operative and conservative treatment for stress-positive SER IV equivalent fractures. Of concern was the fact that 8 patients $(20 \%)$ in the conservative group developed a malalignment and that 8 patients developed union problems. Similarly, in 2016, Willett et al. ${ }^{27)}$ published excellent functional results of conservative treatment for unstable ankle fractures in patients older than 60 years. But, $15 \%$ of the casting group displayed radiographic malunion as compared with $3 \%$ who underwent surgery. These 2 studies demonstrated only short- 
Kim et al. Value of Ultrasound for Stability Assessment of Isolated Lateral Malleolar Fractures

Clinics in Orthopedic Surgery • Vol. 12, No. 4, $2020 \bullet$ www.ecios.org

term follow-up (1 year and 6 months, respectively) results. As previous studies have demonstrated the negative effects of malalignment on the contact area, contact pressures, and the development of arthrosis, short-term assessments are unlikely to predict long-term functional outcomes. ${ }^{28,29)}$

To the best of our knowledge, this is the first study that compares US findings to those of arthroscopy for patients with isolated lateral malleolar fractures. Our results confirmed that all patients had complete or partial deltoid ligament rupture surgically. However, only 7 patients had a complete rupture of the deltoid ligament on US. These findings matched US findings.

This study demonstrated that simple and stress radiographic examinations had poor sensitivity and specificity to assess the stability of isolated lateral malleolar fractures. However, US appeared to be more accurate for assessing complete deltoid ligament tears. An inherent limitation of this study was the small number of complete deltoid ligament tears. However, the prevalence of complete tears in this study was similar to that reported in previous studies. ${ }^{9,26}$ In addition, clinical information after treatment was not presented. Surgical or nonsurgical treatment should have been decided by US findings to evaluate the clinical results of treatment protocol using the US as in the study of Koval et al. ${ }^{9)}$ However, the objective this study was to compare radiography, US, and arthroscopy for the diagnosis of unstable ankle fractures. A clinical study needs to be conducted in the near future. Our study lacks MRI information. However, MRI scan poses big financial burden on patients. In addition, the focus of this study was to compare arthroscopy and US, not MRI. US is often criticized for its operator dependency. In this study, a single radiologist who had great experience of diagnostic foot and ankle US performed and interpreted US images.
Therefore, interobserver reliability could not be calculated. Even though this study has some limitations, this was the first study that compared US findings and arthroscopy for patients with isolated lateral malleolar fractures. This study demonstrated that US was accurate for detecting ligament injuries in isolated lateral malleolar fractures. Tears of the deep deltoid ligaments could be diagnosed on US with high accuracy. Therefore, US could be used as a primary or an adjunctive diagnostic tool to assess the stability of isolated lateral malleolar fractures.

\section{CONFLICT OF INTEREST}

No potential conflict of interest relevant to this article was reported.

\section{ACKNOWLEDGEMENTS}

This study was reported in 2017 American Orthopedic Foot and Ankle Society (AOFAS) summer meeting. The abstract was published in Foot and Ankle Orthopedics on September 18, 2017, as part of 2017 AOFAS Annual Meeting Abstracts.

We would like to express deep gratitude to Professor Sunghoon Park (Professor, Department of Radiology, Ajou University School of Medicine), an experienced musculoskeletal radiologist, for enthusiastic encouragement and useful critiques of this research work. We would also like to thank many orthopedic residents of Ajou University Hospital including Dr. Seong-Yeon Lim (Clinical fellow, Department of Orthopedic Surgery, Ajou University School of Medicine) for their support in the radiographic analysis.

\section{REFERENCES}

1. Nortunen S, Lepojarvi S, Savola O, et al. Stability assessment of the ankle mortise in supination-external rotation-type ankle fractures: lack of additional diagnostic value of MRI. J Bone Joint Surg Am. 2014;96(22):1855-62.

2. Park SS, Kubiak EN, Egol KA, Kummer F, Koval KJ. Stress radiographs after ankle fracture: the effect of ankle position and deltoid ligament status on medial clear space measurements. J Orthop Trauma. 2006;20(1):11-8.

3. Sanders DW, Tieszer C, Corbett B; Canadian Orthopedic Trauma Society. Operative versus nonoperative treatment of unstable lateral malleolar fractures: a randomized multicenter trial. J Orthop Trauma. 2012;26:129-34.
4. Slobogean GP, Marra CA, Sadatsafavi M, Sanders DW; Canadian Orthopedic Trauma Society. Is surgical fixation for stress-positive unstable ankle fractures cost effective? Results of a multicenter randomized control trial. J Orthop Trauma. 2012;26(11):652-8.

5. Tornetta P 3rd. Competence of the deltoid ligament in bimalleolar ankle fractures after medial malleolar fixation. J Bone Joint Surg Am. 2000;82(6):843-8.

6. van den Bekerom MP, Mutsaerts EL, van Dijk CN. Evaluation of the integrity of the deltoid ligament in supination external rotation ankle fractures: a systematic review of the literature. Arch Orthop Trauma Surg. 2009;129(2):227-35. 
Kim et al. Value of Ultrasound for Stability Assessment of Isolated Lateral Malleolar Fractures

Clinics in Orthopedic Surgery • Vol. 12, No. 4, $2020 \bullet$ www.ecios.org

7. Coughlin MJ, Saltzman CL, Anderson RB. Mann's surgery of the foot and ankle. 9th ed. Philadelphia: Saunders/Elsevier; 2013.

8. Joy G, Patzakis MJ, Harvey JP Jr. Precise evaluation of the reduction of severe ankle fractures. J Bone Joint Surg Am. 1974;56(5):979-93.

9. Koval KJ, Egol KA, Cheung Y, Goodwin DW, Spratt KF. Does a positive ankle stress test indicate the need for operative treatment after lateral malleolus fracture? A preliminary report. J Orthop Trauma. 2007;21(7):449-55.

10. Nortunen S, Flinkkila T, Lantto I, et al. Diagnostic accuracy of the gravity stress test and clinical signs in cases of isolated supination-external rotation-type lateral malleolar fractures. Bone Joint J. 2015;97(8):1126-31.

11. Schuberth JM, Collman DR, Rush SM, Ford LA. Deltoid ligament integrity in lateral malleolar fractures: a comparative analysis of arthroscopic and radiographic assessments. J Foot Ankle Surg. 2004;43(1):20-9.

12. Cheung Y, Perrich KD, Gui J, Koval KJ, Goodwin DW. MRI of isolated distal fibular fractures with widened medial clear space on stressed radiographs: which ligaments are interrupted? AJR Am J Roentgenol. 2009;192(1):W7-12.

13. Kemmochi M, Sasaki S, Fujisaki K, Oguri Y, Kotani A, Ichimura S. A new classification of anterior talofibular ligament injuries based on ultrasonography findings. J Orthop Sci. 2016;21(6):770-8.

14. Chen PY, Wang TG, Wang CL. Ultrasonographic examination of the deltoid ligament in bimalleolar equivalent fractures. Foot Ankle Int. 2008;29(9):883-6.

15. Gill JB, Risko T, Raducan V, Grimes JS, Schutt RC Jr. Comparison of manual and gravity stress radiographs for the evaluation of supination-external rotation fibular fractures. J Bone Joint Surg Am. 2007;89(5):994-9.

16. Schock HJ, Pinzur M, Manion L, Stover M. The use of gravity or manual-stress radiographs in the assessment of supination-external rotation fractures of the ankle. J Bone Joint Surg Br. 2007;89(8):1055-9.

17. Michelson JD, Varner KE, Checcone M. Diagnosing deltoid injury in ankle fractures: the gravity stress view. Clin Orthop Relat Res. 2001;(387):178-82.

18. Michelson JD. Ankle fractures resulting from rotational in- juries. J Am Acad Orthop Surg. 2003;11(6):403-12.

19. Egol KA, Amirtharajah M, Tejwani NC, Capla EL, Koval KJ. Ankle stress test for predicting the need for surgical fixation of isolated fibular fractures. J Bone Joint Surg Am. 2004;86(11):2393-8.

20. McConnell T, Creevy W, Tornetta P 3rd. Stress examination of supination external rotation-type fibular fractures. J Bone Joint Surg Am. 2004;86(10):2171-8.

21. Petrisor BA, Poolman R, Koval K, Tornetta P 3rd, Bhandari M; Evidence-Based Orthopaedic Trauma Working Group. Management of displaced ankle fractures. J Orthop Trauma. 2006;20(7):515-8.

22. Gardner MJ, Demetrakopoulos D, Briggs SM, Helfet DL, Lorich DG. The ability of the Lauge-Hansen classification to predict ligament injury and mechanism in ankle fractures: an MRI study. J Orthop Trauma. 2006;20(4):267-72.

23. Nielson JH, Sallis JG, Potter HG, Helfet DL, Lorich DG. Correlation of interosseous membrane tears to the level of the fibular fracture. J Orthop Trauma. 2004;18(2):68-74.

24. Vogl TJ, Hochmuth K, Diebold T, et al. Magnetic resonance imaging in the diagnosis of acute injured distal tibiofibular syndesmosis. Invest Radiol. 1997;32(7):401-9.

25. Cha SD, Kwak JY, Gwak HC, et al. Arthroscopic assessment of intra-articular lesion after surgery for rotational ankle fracture. Clin Orthop Surg. 2015;7(4):490-6.

26. Henari S, Banks LN, Radovanovic I, Queally J, Morris S. Ultrasonography as a diagnostic tool in assessing deltoid ligament injury in supination external rotation fractures of the ankle. Orthopedics. 2011;34(10):e639-43.

27. Willett K, Keene DJ, Mistry D, et al. Close contact casting vs surgery for initial treatment of unstable ankle fractures in older adults: a randomized clinical trial. JAMA. 2016;316(14):1455-63.

28. Kwon JY, Cronin P, Velasco B, Chiodo C. Evaluation and significance of mortise instability in supination external rotation fibula fractures: a review article. Foot Ankle Int. 2018;39(7):865-73.

29. Ramsey PL, Hamilton W. Changes in tibiotalar area of contact caused by lateral talar shift. J Bone Joint Surg Am. 1976;58(3):356-7. 